\title{
When Plans Distinguish Bayes Nets
}

\author{
Alex Dekhtyar \\ Judy Goldsmith \\ Janice L. Pearce
}

\begin{abstract}
We consider the complexity of determining whether differing probability distributions for the same Bayes net result in different policies, significantly different policy outcomes or optimal value functions.
\end{abstract}

\section{Introduction}

One of the classic AI problems is that of planning ${ }^{8}$. In recent years, planning has moved from Blocks World and other toy domains to complex, realistic, and sometimes mission-critical, scenarios. With this move has come the need, in these uncertain days, to solve planning problems for stochastic systems.

Whether the system being modeled is a medical scenario or a military one, a social-service provider network or a university, or a simple vacation ranch with horse riding, there are uncertainties about the effects of a controller's actions. These uncertainties can be modeled as probability distributions over possible outcomes. The controller's choices are guided by knowledge of goals or utilities; the controller's job is to optimize the expected outcome.

One can model a controlled stochastic system as a Markov decision process: a set of states, a set of actions and their stochastic outcomes, and the utilities associated 
with the states and actions. A planning problem consists of such a model, plus the time the system will run, and the optimization criterion: total expected reward, discounted expected reward, etc. However, one can also refer to the model itself as a planning problem, if time horizon and evaluation criterion defaults exist.

In many of the settings mentioned above, the Markov decision process models are enormous. One way to manage huge state spaces is to factor them, either by expressing them in a STRIPS-like model ${ }^{8}$ or as a Bayes net ${ }^{26}$.

A plan or policy for a Markov decision process (MDP) is a function mapping states to actions. While some have considered stochastic functions, we assume here that all functions are deterministic, that is, a planning algorithm outputs the same policy each time it is applied to a given MDP. Papadimitriou and Tsitsiklis showed that, for infinite horizon policies, the problem of finding an optimal policy is in $\mathrm{P}$ 24 . It is not known what the complexity is for the finite horizon, but it is between $\mathrm{P}$ and NP.

When the domain is represented as a Bayes net, the size of the model might shrink logarithmically. This does not, however, lead to a corresponding improvement in complexity, nor does it necessarily cause the size of the actual policies to shrink. The problem of finding an optimal policy for an MDP in the Bayes net model is exponential-time hard ${ }^{23}$. Note that the translation from MDP to Bayes net is usually via a dynamic Bayes net, which explicitly represents time steps. However, in this paper, we do not restrict our consideration to dynamic Bayes nets. The results apply to either the general Bayes net model or the dynamic Bayes net model.

The problems considered in this paper arise in the process of building Bayes net models of real-world planning domains. Consider the question: Where do the numbers come from? There are algorithms for learning conditional probabilities from data, and there are techniques for eliciting probabilities from experts. Suppose that two experts are consulted, or two sets of data, or that an expert's predictions do not match those learned from data. It is not always clear how to combine differing conditional probabilities.

The problem of combining knowledge from different sources in probabilistic models (also known as the information fusion problem) ${ }^{5}$ is a challenging one. If, for instance, the probabilities come from independent data sources of known sizes, one can take an average of the probabilities, weighted by the size of the sample ${ }^{29,22}$. However, if the relative importance or reliability of sources is not known, it is more difficult to choose weights. The problem of fusion of interval probabilities - an apparently easier problem, since intervals can be combined via union or intersection - is addressed by Ferson, et al. ${ }^{7}$; in that work, the authors discuss the various properties different amalgamation or fusion methods might have. None are shown to be optimal for all circumstances.

In the best of all possible worlds, if one knew that the two sets of conditional probabilities led to the same policies, the differences in the conditional probabilities would not matter. If it were easy to determine this, then one could circumvent the fusion problem in such cases. 
The problem addressed in this paper is to determine whether a particular planning algorithm, and two different sets of conditional probability distributions for a Bayes net, yield equivalent policies.

We consider two different notions of equivalence for policies. Given a particular planning algorithm, and two different sets of conditional probability distributions for a Bayes net, we get two policies. We ask whether those policies:

- are the same;

- have the same expected rewards or probabilties of success.

Note that we are assuming that the planning algorithms run in polynomial time. Implicit in that assumption is that they do not necessarily find optimal policies. Thus, the planning algorithm must be a component of the input in the complexitytheoretic problem. As it happens, the proofs given in this paper also give hardness results for optimal policies.

We show that the problem of determining, for a given (polynomial-time) planning algorithm and two Bayes nets for the same dependency graph, whether the policies produced are the same is coNP-complete.

We make the simplifying assumption that policy evaluation is in P. Given this, we show that determining, for a given (polynomial-time) planning algorithm and two Bayes nets for the same dependency graph, whether the policies produced have the same expectation of success or the same expected reward is coNP ${ }^{\mathrm{PP}}$-complete.

Note that the brute-force solution to these problems is to apply the planning algorithm directly to both Bayes nets and compare the policies and their expected outcomes. However, the policies themselves could be exponential in the size of the model, the time needed to find them could be equally large, and the time needed to simply evaluate them might also be exponential. We ask, is there a faster method?

If $\mathrm{P} \neq \mathrm{NP}$, these problems are not in $\mathrm{P}$. However, showing that problems are NP- or coNP-complete immediately indicates the possibility of applying well-studied heuristics. In fact, showing that a problem is $\mathrm{NP}^{\mathrm{PP}}$ or coNP $^{\mathrm{PP}}$-complete also leads to the consideration of heuristics $20,21,19$.

\section{Definitions}

In this section, we formally define Bayes net representations of planning problems, and planning algorithms.

Definition 1. Let $V=\left\{v_{1}, \ldots, v_{n}\right\}$ be a set of random variables with domains $\operatorname{dom}\left(v_{1}\right), \ldots, \operatorname{dom}\left(v_{n}\right)$ respectively, all finite.

A Bayes net $B$ consists of a directed acyclic graph (DAG) $G=(V, E)$, and a set of conditional probability distribution functions $P_{v}: \operatorname{dom}($ parents $(v)) \times \operatorname{dom}(v) \rightarrow$ $[0,1]$ for each $v \in V$, such that for each set of values of parents $(v), P_{v}$ defines a probability distribution over $\operatorname{dom}(v)$.

A dynamic Bayes net or two-phase temporal Bayes net is a Bayes net on a 
directed, acyclic bipartite a graph $G=\left(V, V^{\prime}, E\right)$, where $|V|=\left|V^{\prime}\right|$ and the nodes in $V$ have in-degree 0 . The nodes in $V^{\prime}$ represent the same state variables as those in $V$, but at a subsequent time step. This allows us to address the frame problem: How does a node's current state affect its next state? It also allows us to explicitly model Markov decision processes.

A total state $s$ of $B$ is a total function $s: V \rightarrow \cup_{i=1}^{n} \operatorname{dom}\left(v_{i}\right)$ such that $s\left(v_{i}\right) \in$ $\operatorname{dom}\left(v_{i}\right)$, for all $1 \leq i \leq n$. Let $\mathcal{S}$ be the set of all possible states of the system.

A partial state $s^{\prime}$ of $B$ is a partial function $s^{\prime}: V \rightarrow \cup_{i=1}^{n} \operatorname{dom}\left(v_{i}\right)$ such that $s^{\prime}\left(v_{i}\right) \in \operatorname{dom}\left(v_{i}\right)$, for all $1 \leq i \leq n$.

We often abuse terminology by referring to both total and partial states as simply states. Note that $|\mathcal{S}|$ is generally exponentially larger than the number of random variables $|V|$.

We can interpret a function $P_{v}$ as a probabilistic function from the values of parents $(v)$ to the values of $v$. In this interpretation, the collection of $P_{v}$ s models the probabilistic evolution of states of the Bayes net.

The most common use of Bayes nets is for inference: given a set of values of some nodes, compute the most likely values of other nodes (either parents or children). However, we are interested in the use of Bayes Nets for planning.

We consider the extension of Bayes net models that allows us not only to model the probabilistic evolution of the system, but also to model an outside controller that can affect that evolution. As the means of modelling possible effects of an outside controller on the system, we employ actions, defined formally below.

Definition 2. Let $G=(V, E)$ be a directed acyclic graph. An action $a$ on $G$ is a set of conditional probability distribution functions $P_{a, v}: \operatorname{dom}($ parents $(v)) \times \operatorname{dom}(v) \rightarrow$ $[0,1]$ for each $v \in V$.

A Bayes net representation of a planning domain $B$ is a tuple $\langle G=(V, E), A, \mathcal{F}\rangle$, where $G$ is a DAG, $A$ is a set of stochastic actions on $G$ and $\mathcal{F}$ is a set of states called goal states ${ }^{\mathrm{b}}$.

A Bayes net may also model a Markov decision process, by including an explicit utility function $r: \mathcal{S} \rightarrow \mathbf{R}$ instead of $\mathcal{F}$.

While simple Bayes nets are useful in modelling situations for which we have no control, Bayes net planning domains are useful when there is an opportunity to affect the outcome of a process. For the rest of this paper, we will use "Bayes net" to refer to a Bayes net planning domains. Note that the initial definition of

${ }^{a}$ Generally speaking, dynamic Bayes nets need not have bipartite graphs. However, as Littman showed $^{17}$ that for any Bayes net with synchronous arcs there is an equivalent Bayes net with asynchronous arcs, we assume that dynamic Bayes nets do have bipartite graphs.

b One can easily convert a goal state Bayes net to a utility based Bayes net. For the purposes of later theorems it is easier to use the goal-state formalism. This does not increase the complexity of the computational problems considered. 
a Bayes net corresponds to a Bayes net in the extended definition with only one action, where $\mathcal{F}$ is the set of all states.

We describe the set of choices a controller makes as a policy. For the purposes of this paper, we consider the simplest form of policies, namely mappings from states to actions. This imposes consistency on the controller's choices.

Definition 3. Given a Bayes net $B$, a policy for $B$ is a mapping $\pi: \mathcal{S} \rightarrow \mathcal{A}$.

A planning algorithm $\mathcal{A}$ is a function that maps Bayes nets to plans for those Bayes nets.

A policy can be represented as an explicit list or table of values, as a function specified by some computational device, or implicitly by specifying its values on subsets of the domain, and giving a combining function. We assume that the planning algorithm and the Bayes net themselves form an implicit representation of the policy; when they and a state of the system are specified, we can easily determine the action for that policy.

Other definitions of policies exist in the literature, including time-dependent and history-dependent policies. The hardness proofs in this paper can be easily modified to give the same complexity results for time-dependent policies. (These two notions differ if the underlying system is not fully observable.)

One can see how good a particular planning algorithm is by evaluating the policies that it produces. There are at least two ways to evaluate a policy: goalstate-oriented and reward-oriented. If the former is used, the probability of reaching a goal state following the policy is used as the key evaluating factor. The second way assumes that certain rewards, which accumulate over time, are associated with taking actions in different states of the system. The total accumulated reward over time serves as the evaluation criterion in this case. Thus, the task of a controller is to maximize the probability of reaching a goal state. Note that, in many cases, the optimization criterion is a discounted expected reward: All rewards at future steps are discounted by a factor of $\gamma \in[0,1]$.

Note that the problem of finding an optimal policy for general Bayes nets is EXP-complete ${ }^{17}$. However, for this paper, we assume that the planning algorithms are tractable. This implies either that the Bayes nets fall into some special subclass, or that the planning algorithms are approximate. In particular, we often assume planning algorithms $\mathcal{A}$ such that, for any Bayes net $B$ and state $s$ of $B$, computing the action $\mathcal{A}(B)(s)$ can be done in time polynomial in $|B|$.

\section{Example}

Consider the following example which features a small (fictitious) horseback riding summer resort. The resort consists of a number of trails and a stable compound which attract horse owners for recreational purposes. The owners of the resort are interested in maximizing their profit, which is determined by the rates they charge the riders for the use of their stables and trails as well as by the number of riders 


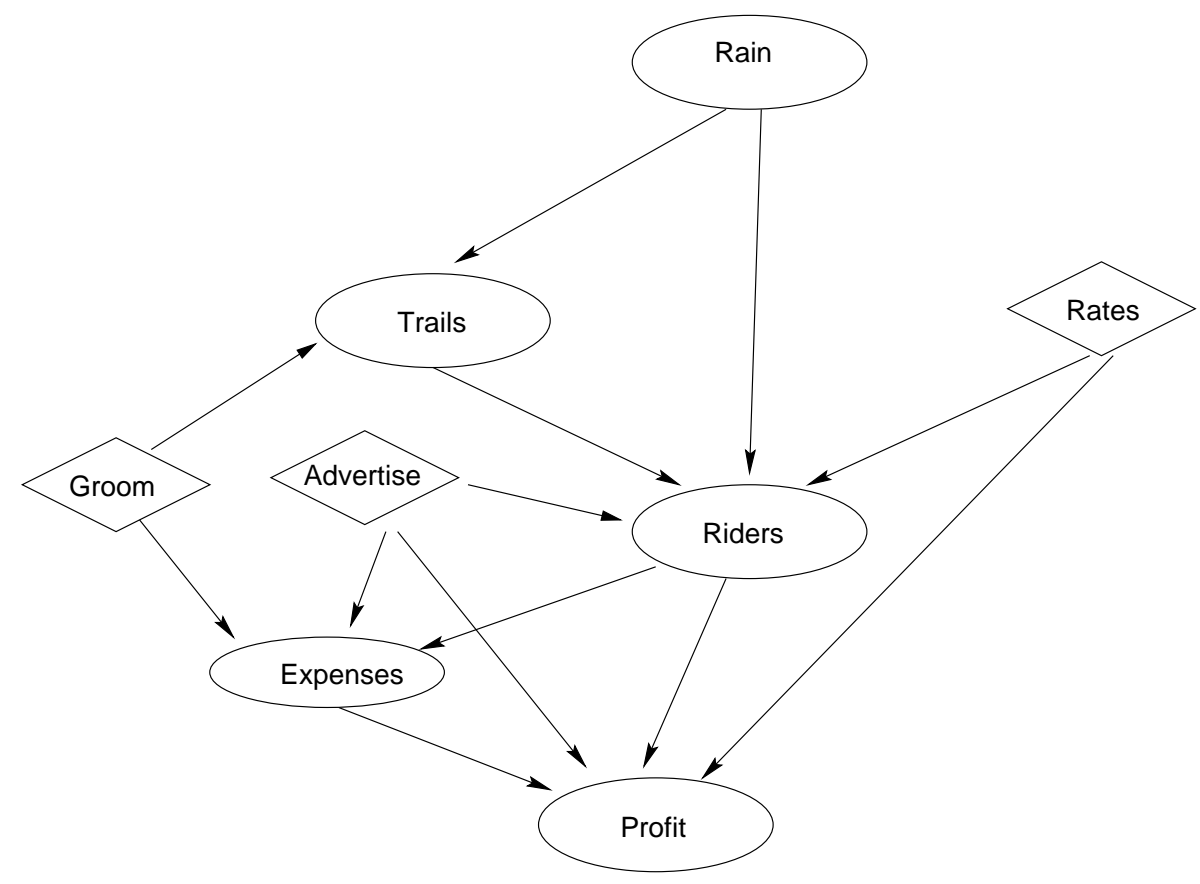

Fig. 1. Sample Bayes net: Horseback Riding Resort

that visit the resort over a period of time. The profit is also adversely affected by the expenses that the owners incur. In this simple example, we assume that the number of riders visiting the resort is influenced by the weather conditions, state of the trails, and by the current trail and stable rates. The owners may choose to spend the money on advertising the resort, and on grooming trails: each action possibly resulting in an increased number of visitors.

This situation can formally be represented as the Bayes net depicted in Figure 1. Two types of variables are present in the network: random variables and action variables, the latter drawn as diamonds in the Figure. The values of random variables are determined stochastically based on the values of other variables in the network. The values of action variables are set by the actions (decisions) of resort owners and thus are deterministic. The random variables are:

- rain: the presence or absence of rain or other inclement weather;

- trails: fair or poor condition of trails;

- riders: high or low ridership on trails;

- expenses: high or low expenses to support the resort operation;

- profit: at the end of the period, the resort can turn in low or high profit.

The action variables are: 


\begin{tabular}{llllllll}
\multicolumn{2}{l}{$\begin{array}{l}\text { Node: rain } \\
P(\text { rain }=\text { yes })\end{array}$} & & & \multicolumn{4}{c}{$P($ trails $=$ fair $)$} \\
Set 1 & Set 2 & Set 3 & groom & rain & Set 1 & Set 2 & Set 3 \\
0.2 & 0.2 & 0.2 & no & no & 0.6 & 0.6 & 0.6 \\
& & & no & yes & 0.3 & 0.3 & 0.3 \\
& & yes & no & 1.0 & 1.0 & 1.0 \\
& & & yes & yes & 0.8 & 0.8 & 0.8
\end{tabular}

Node: expenses

\begin{tabular}{llllll} 
& & & \multicolumn{3}{c}{$P($ expenses $=$ high $)$} \\
groom & advertise & riders & Set 1 & Set 2 & Set 3 \\
no & no & low & 0.35 & 0.35 & 0.35 \\
no & no & high & 0.5 & 0.5 & 0.5 \\
no & yes & low & 0.5 & 0.5 & 0.5 \\
no & yes & high & 0.65 & 0.65 & 0.65 \\
yes & no & low & 0.45 & 0.45 & 0.45 \\
yes & no & high & 0.6 & 0.6 & 0.6 \\
yes & yes & low & 0.65 & 0.65 & 0.65 \\
yes & yes & high & 0.75 & 0.75 & 0.75
\end{tabular}

Table 1. Sets of Conditional Probability Distributions for Horseback Riding Resort Bayes Nets (Part I).

- rates: high or low rates charged for the use of the stable and trails;

- groom: indicates whether the trails are groomed or not.

- advertise: indicates whether the resort is advertising to the potential visitors or not.

All variables are binary for simplicity ${ }^{\mathrm{c}}$. As mentioned above, the owners of the resort can take certain actions in order to affect the situation with the goal of maximizing the probability of turning high profit. These actions are represented in the model as the assignment of values to the action variables rates, groom and advertise. The actions affect the conditional probability distributions associated with certain nodes of the Bayes net. An atomic action is the assignment of a value to a single action variable. Values to different action variables are assigned independently, thus there are eight compound actions that the owners may choose. The effects of atomic actions are described in Table 3. Basically, each atomic action produces one positive and one negative effect. Compound actions produce overlapping effects: e.g., advertising and grooming trails result in a high probability of incurring high expenses

${ }^{c}$ The SPUDD planning algorithm ${ }^{13,11}$ that we have used in our example requires binary variables. 
Node: riders

\begin{tabular}{lllllll} 
& & & & \multicolumn{3}{c}{$P($ riders $=$ high $)$} \\
rates & advertise & trails & rain & Set 1 & Set 2 & Set 3 \\
low & no & poor & no & 0.5 & 0.5 & 0.5 \\
low & no & poor & yes & $\mathbf{0 . 3}$ & $\mathbf{0 . 3 5}$ & 0.3 \\
low & no & fair & no & $\mathbf{0 . 9}$ & $\mathbf{0 . 8 5}$ & 0.9 \\
low & no & fair & yes & $\mathbf{0 . 6}$ & $\mathbf{0 . 5 5}$ & 0.6 \\
low & yes & poor & no & $\mathbf{0 . 9}$ & $\mathbf{0 . 8 5}$ & 0.9 \\
low & yes & poor & yes & $\mathbf{0 . 6 5}$ & $\mathbf{0 . 7}$ & $\mathbf{0 . 7 5}$ \\
low & yes & fair & no & $\mathbf{0 . 9 5}$ & $\mathbf{0 . 9}$ & 0.95 \\
low & yes & fair & yes & $\mathbf{0 . 8}$ & $\mathbf{0 . 7 5}$ & 0.8 \\
high & no & poor & no & $\mathbf{0 . 2 5}$ & $\mathbf{0 . 3}$ & 0.25 \\
high & no & poor & yes & $\mathbf{0 . 2}$ & $\mathbf{0 . 2 5}$ & 0.2 \\
high & no & fair & no & 0.7 & 0.7 & 0.7 \\
high & no & fair & yes & 0.4 & 0.4 & 0.4 \\
high & yes & poor & no & $\mathbf{0 . 3 5}$ & $\mathbf{0 . 3}$ & 0.35 \\
high & yes & poor & yes & 0.3 & 0.3 & 0.3 \\
high & yes & fair & no & 0.8 & 0.8 & 0.8 \\
high & yes & fair & yes & 0.5 & 0.5 & 0.5
\end{tabular}

Node: profits

\begin{tabular}{lllllll} 
& & & & \multicolumn{3}{c}{$P($ profits $=$ high) } \\
rates & advertise & expenses & riders & Set 1 & Set 2 & Set 3 \\
low & no & low & low & $\mathbf{0 . 4 5}$ & 0.45 & $\mathbf{0 . 4}$ \\
low & no & low & high & $\mathbf{0 . 6 5}$ & 0.65 & $\mathbf{0 . 6}$ \\
low & no & high & low & $\mathbf{0 . 3 5}$ & 0.35 & $\mathbf{0 . 3}$ \\
low & no & high & high & $\mathbf{0 . 4 5}$ & 0.45 & $\mathbf{0 . 4}$ \\
low & yes & low & low & $\mathbf{0 . 4 5}$ & 0.45 & $\mathbf{0 . 4}$ \\
low & yes & low & high & $\mathbf{0 . 6 5}$ & 0.65 & $\mathbf{0 . 6}$ \\
low & yes & high & low & $\mathbf{0 . 3 5}$ & 0.35 & $\mathbf{0 . 3}$ \\
low & yes & high & high & $\mathbf{0 . 5 5}$ & 0.55 & $\mathbf{0 . 5}$ \\
high & no & low & low & $\mathbf{0 . 5 5}$ & 0.55 & $\mathbf{0 . 6 5}$ \\
high & no & low & high & $\mathbf{0 . 7 5}$ & 0.75 & $\mathbf{0 . 8 5}$ \\
high & no & high & low & $\mathbf{0 . 4}$ & 0.4 & $\mathbf{0 . 4 5}$ \\
high & no & high & high & $\mathbf{0 . 5}$ & 0.5 & $\mathbf{0 . 5 5}$ \\
high & yes & low & low & $\mathbf{0 . 5 5}$ & 0.55 & $\mathbf{0 . 6 5}$ \\
high & yes & low & high & $\mathbf{0 . 7 5}$ & 0.75 & $\mathbf{0 . 8 5}$ \\
high & yes & high & low & $\mathbf{0 . 4}$ & 0.4 & $\mathbf{0 . 4 5}$ \\
high & yes & high & high & $\mathbf{0 . 6}$ & 0.6 & $\mathbf{0 . 6 5}$
\end{tabular}

Table 2. Sets of Conditional Probability Distributions for Horseback Riding Resort Bayes Nets (Part II).

but also in a high probability of attracting high ridership (both probabilities higher than those for individual actions) as well as in trails being in fair condition.

We have considered three different sets of conditional probability assignments associated with the Bayes net in Figure 1, which we will call Set 1, Set 2 and Set 3 CPTs. Tables 3 and 3 show all three sets of CPTs. The differences of Sets 2 and 3 from Set 1 are highlighted in boldface.

For our planning algorithm we have used SPUDD, an on-line system for planning 


\begin{abstract}
Action
Implications

Rates

Charge low rates Increased probability of high ridership

Decreased probability of high profit

Charge high rates Increased probability of high profits

Decreased probability of high ridership

Groom

Do not groom trails Decreased probability of fair condition of the trails

Decreased probability of high expenses

Groom trails Increased probability of fair condition of the trails

Increased probability of high expenses
\end{abstract}

Advertise

Do not advertise

Decreased probability of high ridership

Decreased probability of high expenses

Advertise Increased probability of high ridership

Increased probability of high expenses

Table 3. Implications of Actions.

under uncertainty, developed by Hoey, St.-Aubin, Hu and Boutiler ${ }^{13,11}$, to come up with the optimal policies for maximizing profit in our Horseback Riding Resort domain under each of the three sets of conditional probability tables. ${ }^{\mathrm{d}}$

SPUDD output consists of two decision trees: the optimal policy decision tree and the value function (expected reward) decision tree. Figure 2 illustrates the optimal policies for all three sets of CPTs returned by SPUDD.

We can see that the first two CPTs (Sets 1 and 2) yield the same ppolicy (the decision trees are rendered differently by SPUDD but are isomomorphic), whereas Set 3 yields a drastically different policy. On the other hand, the three value functions are all distinct. (They are not included in the text because the trees are wide enough that they cannot be rendered in readable formats. Interested readers can find the decision trees for the optimal value functions at http://www.cs.uky.edu/ dekhtyar/dblab/fusion.html.)

Careful inspection of the three sets of probability tables might not reveal these outcomes. This is not surprising, given the intractibility of distinguishing Bayes nets, as shown in what follows.

It is not, however, surprising that the same policy applied to Bayes nets with

${ }^{\mathrm{d}}$ SPUDD web page is http://www.cs.ubc.ca/spider/staubin/Spudd/index.html. At http://www.cs.ubc.ca/spider/staubin/Spudd/form.html one can run SPUDD interactively. We have used default SPUDD settings. The data files describing our Horseback Riding Resort example, as well as SPUDD output can be obtained from http://www.cs.uky.edu/ dekhtyar/dblab/fusion.html; the data file for Set 1 is also included in Appendix Appendix A. 


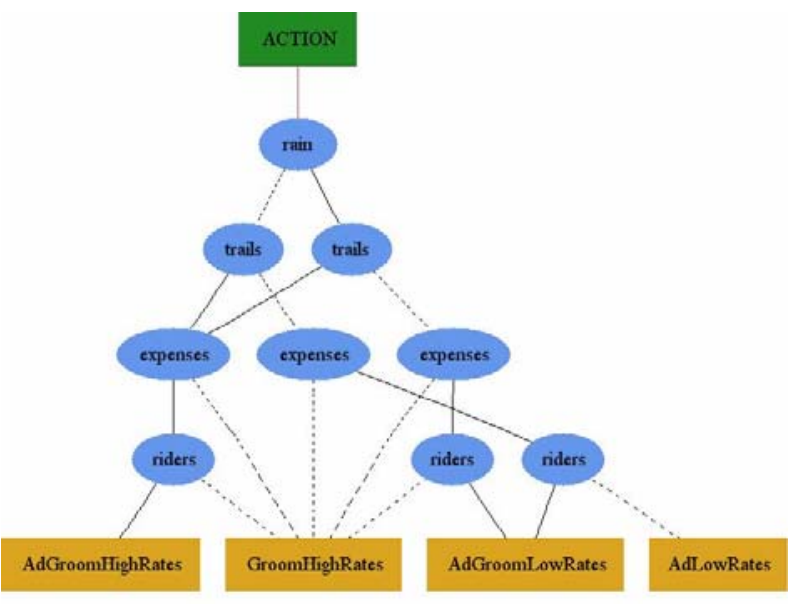

(a)

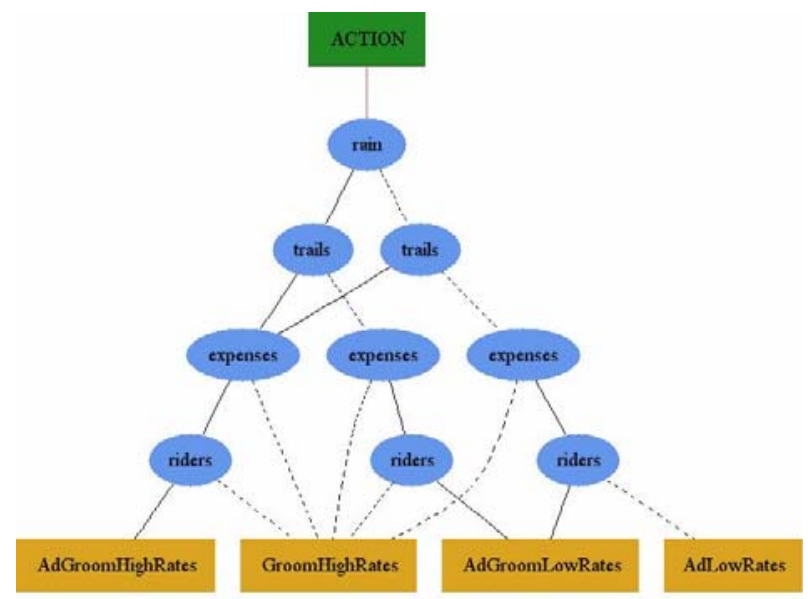

(b)

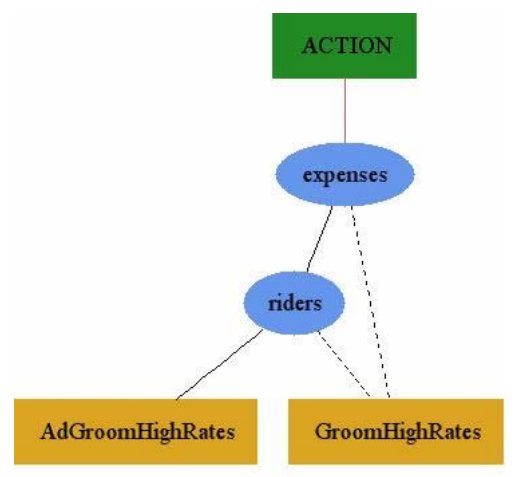

(c)

Fig. 2. Decision trees for SPUDD optimal policies for Sets 1 (a), 2 (b) and Set 3 (c) CPTs in Horseback Riding Resort example. 
different CPTs might yield different value functions. One might expect it to be difficult to find different CPTs that yield the same value function for any but the crudest policies.

It is also interesting to note how three different notions of Bayes net equivalence with respect to planning relate to this example. Suppose the owners ask three different consultants to evaluate the operation of the resort, resulting in Sets 13 , respectively. The owners must make an information fusion decision: choose one set or combine them somehow. What they choose depends on what they want: advice on what actions to take, or expected profit under some pre-determined policy, or perhaps the optimal expected profit. (If they are considering bankruptcy, the bank might for instance be interested in the latter.) These three criteria correspond precisely to the three definitions of Bayes net equivalence under planning discussed in this paper.

Because the example considered here is so small, the problem of finding an optimal policy for it with SPUDD is tractable. Thus, we have used an optimal planning algorithm, so the optimal value and policy value are the same here.

\section{Detecting Identical Policies}

We first consider detecting identical policies.

Definition 4. Let $B_{1}=\left\langle G, P_{1}\right\rangle$ and $B_{2}=\left\langle G, P_{2}\right\rangle$ be two Bayes nets over the same graph $G$. Let $\mathcal{A}$ be some planning algorithm. The triple $\left\langle B_{1}, B_{2}, \mathcal{A}\right\rangle$ is in BNPlan if and only if for every state $s$ of $B_{1}$ and $B_{2}$, e

$$
\mathcal{A}\left(B_{1}, s\right)=\mathcal{A}\left(B_{2}, s\right) .
$$

Intuitively, two Bayes nets over the same structure belong to the class BNPlan together with the planning algorithm $\mathcal{A}$ if the algorithm does not distinguish between the Bayes nets.

When the planning algorithm is fixed, we will denote as $B N \operatorname{Plan}^{\mathcal{A}}$ the set of pairs of Bayes nets $\left\langle B_{1}, B_{2}\right\rangle$ such that for all states $s, \mathcal{A}\left(B_{1}, s\right)=\mathcal{A}\left(B_{2}, s\right)$.

Also, given the graph structure $G$ of a family of Bayes nets $B N_{G}$, we can consider the sets $B N$ Plan $_{G} \subset B N P l a n$ and $B N$ Plan $_{G}^{\mathcal{A}} \subset B N$ Plan $^{\mathcal{A}}$ of Bayes net pairs whose graph structure is $\mathrm{G}$.

First, we study the problem of the complexity of determining that a triple $\left\langle B_{1}, B_{2}, \mathcal{A}\right\rangle$ belongs to class BNPlan. The following results show that this problem is coNP-complete.

Theorem 1. The BNPlan problem is coNP-hard.

Proof. We consider the dual problem of determining $\left\langle B_{1}, B_{2}, \mathcal{A}\right\rangle \notin B N$ Plan and will show that this problem is NP-hard.

e Since both $B_{1}$ and $B_{2}$ are Bayes nets over the same graph, their sets of nodes, and thus of states, coincide. 


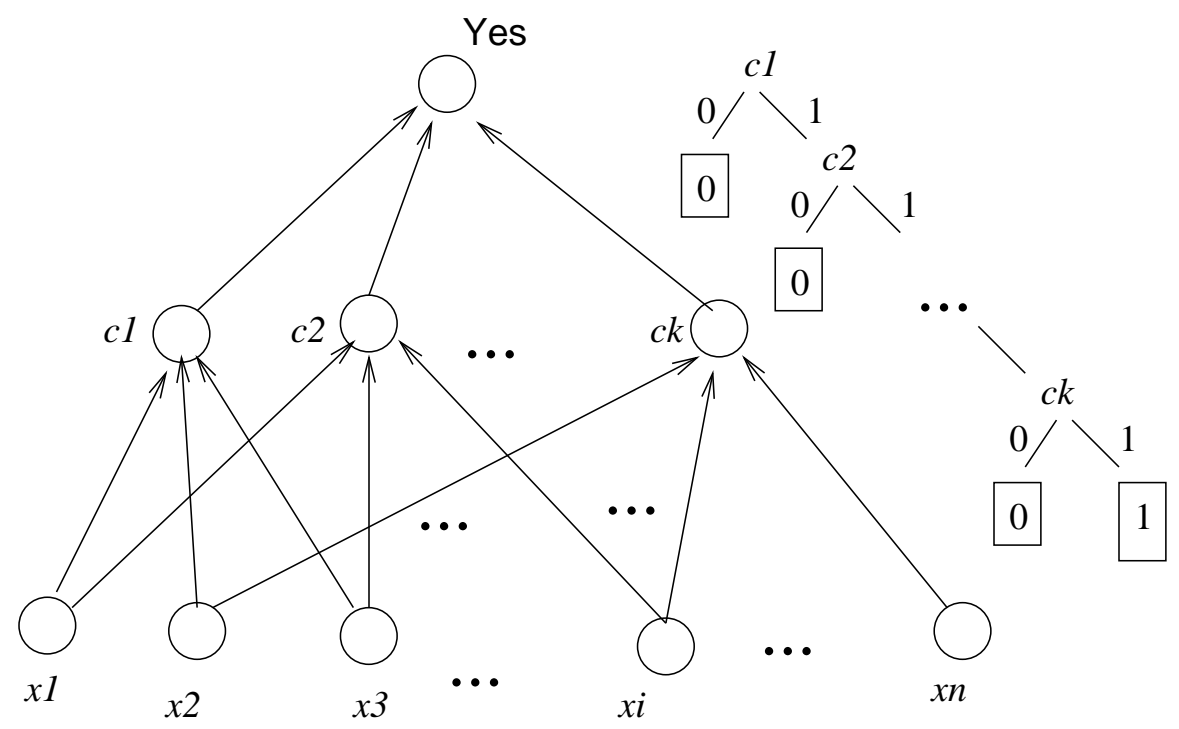

Fig. 3. Bayes net structure for Satisfiability problem and the the decision tree for the Yes node for net $B_{1}$.

Let $\varphi$ be a propositional Boolean formula over variables $x_{1}, \ldots, x_{n}$. Without loss of generality we can consider $\varphi$ to be in 3CNF.

Given $\varphi$, we will construct a pair of Bayes nets $B_{1}^{\varphi}$ and $B_{2}^{\varphi}$ (we will omit the superscripts where this does not generate ambiguity) and a planning algorithm $\mathcal{A}$ such that $\varphi \in S A T$ if and only if $\left\langle B_{1}^{\varphi}, B_{2}^{\varphi}, \mathcal{A}\right\rangle \notin B N$ Plan.

The Bayes net graph structure $G$ underlying both $B_{1}$ and $B_{2}$ is shown in Figure 3 . The net will consist of three levels of nodes which take on the values of 0 and 1 (false and true). At the lowest or initial level, a node will be associated with each of the variables $x_{1}, \ldots, x_{n}$ of $\varphi$. At the second or middle level, the nodes represent the clauses of $\varphi$. Each node $c_{i}$ from the second level is connected to exactly three nodes representing Boolean variables variables.

The third level of the network consists of one node, which we will label as Yes. Its parents will be all second level nodes $c_{1}, \ldots, c_{k}$.

The conditional probability distributions $P_{1}$ associated with the Bayes net $B_{1}$ will faithfully simulate the computation of the truth value of $\varphi$. Each node $x_{1}, \ldots, x_{n}$ is a binary decision node, its value controlled from outside.

For each second level node $c$ with parents $x, x^{\prime}$, and $x^{\prime \prime}$, the conditional probability table will simulate the truth table for the conjunct represented by $c$. E.g, if $c=x \vee \neg x^{\prime} \vee x^{\prime \prime}$ the conditional probability table will be: $P_{1}\left(c=0 \mid x=0, x^{\prime}=\right.$ $\left.1, x^{\prime \prime}=0\right)=1 ; P_{1}\left(c=1 \mid x=0, x^{\prime}=1, x^{\prime \prime}=0\right)=0$ (this is the only combination that makes $c$ false $)$ and $P_{1}\left(c=0 \mid x=a, x^{\prime}=a^{\prime}, x^{\prime \prime}=a^{\prime \prime}\right)=0 ; P_{1}\left(c=1 \mid x=a, x^{\prime}=\right.$ $\left.a^{\prime}, x^{\prime \prime}=a^{\prime \prime}\right)=0$ for any other combination of truth values for $x, x^{\prime}$, and $x^{\prime \prime}$. 
Finally, the probability distribution for the node Yes is defined as $P_{1}$ (Yes $=$ $\left.1 \mid c_{1}=1, \ldots, c_{k}=1\right)=1 ; P_{1}\left(\right.$ Yes $\left.=0 \mid c_{1}=1, \ldots, c_{k}=1\right)=0$ and $P_{1}\left(\right.$ Yes $=1 \mid c_{1}=$ $\left.a_{1}, \ldots, c_{k}=a_{k}\right)=0 ; P_{1}\left(\mathrm{Yes}=0 \mid c_{1}=a_{1}, \ldots, c_{k}=a_{k}\right)=1$ for all other combinations of values of $c_{1}, \ldots, c_{k}$. This distribution, represented explicitely will take $2^{k}$ rows to describe. However, it can be represented compactly, by explicitely specifying only the probability row $P_{1}\left(\right.$ Yes $\left.=1 \mid c_{1}=1, \ldots, c_{k}=1\right)=1$, or, alternatively, by a simple decision tree of size $O(k)$ which checks the values of each node $c_{1}, \ldots, c_{k}$ and outputs 0 anytime it observes a 0 , as depicted on Figure 3 .

The probability distribution tables $P_{2}$ for the Bayes net $B_{2}$ are designed to mimic the probability distributions $P_{1}$ on the lower and middle layer of the nodes. For the node Yes, $P_{2}$ specifies that the probability of it becoming true is always 0 .

The actions associated with these two Bayes nets correspond to setting the values of the lower level nodes (which represent the Boolean variables). The goal states are those with the Yes node set to 1 .

We can now specify the planning algorithm $\mathcal{A}$. Let state $s$ of $B_{1}$ and $B_{2}$ be a sequence $\left(a_{1}, \ldots, a_{n}\right)$ of binary digits representing the truth values of variables $x_{1}, \ldots, x_{n}$. We denote the state of $B_{1}$ and $B_{2}$ which is a lexicographic successor of the binary string $a_{1} a_{2} \ldots a_{n}$ as $\operatorname{succ}(s)$. To make sure that the planning algorithm described below always produces result we set $\operatorname{succ}\left(1^{n}\right)=0^{n}$.

Algorithm $\mathcal{A}$ will leave the state $s$ unchanged if $s$ induces Yes $=1$. Otherwise, it returns the set of actions that change the current state of the $\mathrm{BN} B_{1}$ or $B_{2}$ to the state $\operatorname{succ}(s)$.

We assume that $\mathcal{A}$ is represented either as a Turing machine, or as a program in some agreed-upon programming language. Note that the complexity of BNPlan is measured in terms of $\left|B_{1}\right|+\left|B_{2}\right|+|\mathcal{A}|$, i.e., the size of the representations of the inputs.

In order to see why this construction works we need the following.

(1) The Yes node of $B_{1}$ is set to 1 if and only if the initial state $s$ of $B_{1}$ corresponds to a satisfying assignment to the variables $x_{1}, \ldots, x_{n}$ of the formula $\varphi$.

(2) The Yes node of $B_{2}$ will never be set 1 .

(3) The policies constructed by $\mathcal{A}$ for $B_{1}$ and $B_{2}$ will be identical on all states $s$ unless there is a state $s$ with Yes $=1$.

From items 1 and 3 from the above we conclude that $\left\langle B_{1}^{\varphi}, B_{2}^{\varphi}, \mathcal{A}\right\rangle \notin B N$ Plan if and only if $\varphi \in S A T$.

Theorem 2. The BNPlan $\in$ coNP.

Proof. Notice that, for Bayes net $B, \mathcal{A}(B, s)$ is an action; the size of the representation of actions is $\leq|B|$, since the specification of actions is part of $B$.

To show that $\left\langle B_{1}, B_{2}, \mathcal{A}\right\rangle \notin B N$ Plan, we need only guess state $s$ such that $\mathcal{A}\left(B_{1}, s\right) \neq \mathcal{A}\left(B_{2}, s\right)$. This check is in polynomial time, and the guess is polynomial sized. Thus, BNPlan $\in$ NP, so BNPlan $\in$ coNP. 


\section{Detecting Identical Probabilities of Plan Success}

In the previous section, we considered two Bayes nets to be equivalent, relative to a planning algorithm, if the algorithm produced the same policies. One can relax this definition and ask, does this planning algorithm or policy produce the same outcome, namely, the same probability of success?

We assume here that the specification of the Bayes net and/or the planning algorithm includes the specification of a set of goal states or utility function for each Bayes net. In what follows, we implicitly presume that the sets of goal states for the two Bayes nets in question are the same.

Definition 5. Let $B=\langle G, P\rangle$ be a Bayes net with a given set of goal states. Let $\mathcal{A}$ be a planning algorithm, and $s$ a state of $B$. Then $\operatorname{Succ}(\mathcal{A}, B, s, h)$ is the defined to be the probability that the policy calculated by $\mathcal{A}$, when applied to state $s$ of $B$ and iterated for $h$ steps, will reach a goal state.

Rather than requiring that the success probabilities be identical for the two Bayes nets, we allow an extra parameter to specify how close they must be.

Definition 6. Let $B_{1}=\left\langle G, P_{1}\right\rangle$ and $B_{2}=\left\langle G, P_{2}\right\rangle$ be two Bayes nets over the same graph $G$. Let $\mathcal{A}$ be some planning algorithm. The tuple $\left\langle B_{1}, B_{2}, \mathcal{A}, h, \theta\right\rangle$ is in SBNPlan if and only if for every state $s$ of $B_{1}$ and $B_{2}, \mid \operatorname{Succ}\left(\mathcal{A}, B_{1}, s, h\right)-$ $\operatorname{Succ}\left(\mathcal{A}, B_{2}, s, h\right) \mid \leq \theta$.

Note that we can also consider the value of a Bayes net relative to its optimal policy.

Definition 7. Let $B=\langle G, P\rangle$ be a Bayes net with a given utility function. Let $V_{B}(s, h)$ be the optimal horizon $h$ value function for $B$. Let $B_{1}=\left\langle G, P_{1}\right\rangle$ and $B_{2}=\left\langle G, P_{2}\right\rangle$ be two Bayes nets over the same graph $G$. The tuple $\left\langle B_{1}, B_{2}, h, \theta\right\rangle$ is in $B N$ Value if and only if for every state $s$ of $B_{1}$ and $B_{2},\left|V_{B_{1}}(s, h)-V_{B_{2}}(s, h)\right| \leq \theta$.

Alternatively, one could further quantify SBNPlan over all policies; depending on the complexity of policy descriptions allowed, this might easily increase the complexity of the problem.

Before stating the next theorem, we remind the reader of some facts about the class $\mathrm{NP}^{\mathrm{PP}}$. The class $\mathrm{PP}$ (probabilistic $\mathrm{P}$ ) consists of those languages $L$ for which a non-deterministic polynomial time Turing Machine $\mathrm{N}$ exists such that $x \in L$ if and only if $N(x)$ accepts on more than half of its computations. The class $\mathrm{NP}^{\mathrm{PP}}$ consists of the languages accepted by an NP computation with free access to PP computation. Torán has shown ${ }^{31}$ that $\mathrm{NP}^{\mathrm{PP}}$ languages can be characterized as "guess a proof of polynomial size and verify using a PP computation". The following inclusions show the positions of $\mathrm{PP}$ and $\mathrm{NP}^{\mathrm{PP}}$ with respect to some more well-known complexity classes.

$$
\mathrm{P} \subseteq \mathrm{NP} \subseteq \mathrm{PH} \subseteq \mathrm{PP} \subseteq \mathrm{NP}^{\mathrm{PP}} \subseteq \mathrm{PSPACE} \subseteq \mathrm{EXP} .
$$


The set EMAJSAT is $\leq_{m}^{P}$-complete for $\mathrm{NP}^{\mathrm{PP}}{ }^{18}$. A Boolean formula, natural number pair $\langle\varphi, k\rangle$ is in EMAJSAT if and only if there is a truth assignment to the first $k$ variables of $\varphi$ such that at least half of its completions satisfy $\varphi$.

Theorem 3. The SBNPlan problem is $\mathrm{coNP}^{\mathrm{PP}}$-hard.

Proof. To show this, we give a reduction from EMAJSAT. As in Theorem 1, we consider the dual problem of determining $\left\langle B_{1}, B_{2}, \mathcal{A}, h, \theta\right\rangle \notin S B N P l a n$ and will show that this problem is $\mathrm{NP}^{\mathrm{PP}}$-hard.

Given a pair $\langle\varphi, k\rangle$, we construct an initial Bayes net $B_{1}$ as in the proof of Theorem 1. There are nodes in the Bayes net representing each variable and each clause, and an extra timekeeper node that turns the process off after one step.

Each clause node depends on the variable nodes for those variables it contains. The dependence is deterministic. Variable nodes have no parents. The first $k$ variable nodes are set by the user's action; the remaining variable nodes are set to 1 with probability $1 / 2$, independent of the action chosen or the prior state. The timekeeper node is dependent on all of the clause nodes. It has three states: starting, on and off. The system starts with it in starting. Any action sets it to on; any further actions set it to off. This enforces a horizon of 1.

The goal states are those in which all clause nodes are set to 1 and the timekeeper node is set to on.

In the first Bayes net, $B_{1}$, the clause nodes depend on the variable nodes in the manner indicated by $\varphi$. In the second Bayes net, $B_{2}$, the clause nodes are uniformly set to 0 . Thus, $B_{2}$ can never reach a goal state.

Note that a policy for $B_{1}$ determines a setting of the first $k$ variables. Furthermore, $\langle\varphi, k\rangle \in$ EMAJSAT if and only if there is some policy that reaches a goal state with probability at least $1 / 2$.

Consider the policy $\pi$ which treats the current variable assignment $x_{1}, \ldots, x_{k}$ as a binary string and increments it by one, and maps $1^{k}$ to $0^{k}$. If $\langle\varphi, k\rangle \in$ EMAJSAT then some setting of the first $k$ variables witnesses that. Let $s^{\prime}$ be the lexicographic predecessor of that setting (or let $s^{\prime}=1^{k}$, if that setting is $0^{k}$ ), and let $s$ be any state of the system that extends $s^{\prime}$. Then $\operatorname{Succ}\left(\mathcal{A}, B_{1}, s, 1\right)-\operatorname{Succ}\left(\mathcal{A}, B_{2}, s, 1\right) \geq 1 / 2$.

Thus, $\langle\varphi, k\rangle \in$ EMAJSAT if and only if for some $s, \operatorname{Succ}\left(\mathcal{A}, B_{1}, s, h\right)>{ }_{2}^{1}$ if and only if $\left\langle B_{1}, B_{2}, \mathcal{A}, h,{ }_{2}^{1}\right\rangle \notin S B N$ Plan for any $h \geq 1$.

Corollary 1. The problem BNValue is $\mathrm{coNP}^{\mathrm{PP}}$-hard.

Proof. Consider the construction in the proof of Theorem 3. Note that if $A$ is an optimal policy for $B_{1}$, then, for some $s, \operatorname{Succ}\left(\mathcal{A}, B_{1}, s, 1\right)-\operatorname{Succ}\left(\mathcal{A}, B_{2}, s, 1\right) \geq 1 / 2$ if and only if $\langle\varphi, k\rangle \in$ EMAJS AT. By definition, then, $V_{B_{i}}(s, h)=\operatorname{Succ}\left(\mathcal{A}, B_{i}, s, 1\right)$. Thus, $\langle\varphi, k\rangle \in$ EMAJSAT if and only if $\left\langle B_{1}, B_{2}, h, \theta\right\rangle \in B N V$ Value.

To show coNP ${ }^{\mathrm{PP}}$-completeness, we would need that $S B N P l a n \in \operatorname{coNP}^{\mathrm{PP}}$. This requires that policy evaluation be in PP. Given that assumption, the theorem follows immediately from the appropriate characterization of the class coNP ${ }^{\mathrm{PP}}$. 
In his dissertation, Jacobo Toran ${ }^{31}$ considers the closure of the class $\mathrm{P}$ under polynomially length-bounded existential and universal quantifiers and under the counting quantifier $\mathbf{C}$. If $K$ is a language class, then a language $L$ is in $\mathbf{C} K$ if there is a $B \in K$, an $f \in \mathrm{FP}$, and a polynomial $p$ such that

$$
x \in L \Leftrightarrow \mid\{y:|y| \leq p(|x|) \text { and } B(x, y)\} \mid \geq f(|x|) .
$$

In particular, for $f(x)=1 / 2, \mathrm{PP}=\mathbf{C} K$.

One can define the Counting Hierarchy over P analogously to the Polynomial Hierarchy: it is the closure of $\mathrm{P}$ under polynomially length bounded existential, universal, and counting quantifiers. Toran showed that, for any $K$ in the counting hierarchy, $\mathrm{NP}^{K}=\exists^{P} K$. In particular,

$$
\mathrm{NP}^{\mathrm{PP}}=\exists^{P} \mathrm{PP}=\exists^{P} \mathbf{C P} \text { and } \operatorname{coNP}^{\mathrm{PP}}=\forall^{P} \mathbf{C} P \text {. }
$$

This characterization gives us another method for showing membership in $\mathrm{NP}^{\mathrm{PP}}$ and $\mathrm{coNP}^{\mathrm{PP}}$, in terms of these quantifiers. Corollary 2 follows immediately from this.

\section{Corollary 2.}

(1) If $\operatorname{Succ}(\mathcal{A}, B, s, 1) \in \mathrm{PP}$, then $S B N P l a n \in \operatorname{coNP}^{\mathrm{PP}}$.

(2) If $\operatorname{Val}_{B}(s, h) \in \mathrm{PP}$ then $B N$ Value $\in \mathrm{coNP}^{\mathrm{PP}}$.

\section{Easier Cases}

Although we have shown that, in the general case, it is computationally complex to decide whether two sets of conditional probability tables are equivalent for a Bayes net structure, there are some cases where these worst-case analyses do not apply. It is an open question to characterize other useful categories of Bayes nets and policies, but we give some preliminary thoughts on this.

For our analysis, let $n$ be the number of nodes in the Bayes net in question. We consider Bayes nets where the number of actions available is $\mathcal{O}(n)$. We refer to any number $k=\mathcal{O}(\log n)$ as a small number.

Suppose for some network structure $G, \mathcal{A}$ generates a policy where each action is chosen based on a fixed, small set of nodes. Then for Bayes nets $B_{1}$ and $B_{2}$ over structure $G$, it is easy to determine whether $\left\langle B_{1}, B_{2}, \mathcal{A}\right\rangle \in B N$ Plan: one merely enumerates all possible values of those few nodes and asks whether the policies are equivalent for those values.

For instance, if a policy in our Horseback Riding Resort example (see Section 3 ) is based solely on the number of riders then this case applies. This is perhaps too trivial an example. Imagine, instead, that policies are based on the number of riders and the weather. Then one need not consider the expenses incurred by the resort.

Suppose, instead, that we have a network structure $G$ consisting of a number of disjoint subnetworks, each consisting of a small number of nodes. Suppose, in 
addition, that the function that determines the goal state can be expressed simply, for instance as the conjunction of a small number of independent node values. In this case, the probability of the conjunction is the minimum of the probabilities of achieving each given goal node value. Thus, $\operatorname{Succ}(\mathcal{A}, B, s, h) \geq \theta$ if and only if the probability of "success" for each goal variable is at least $\theta$. To determine this for all $s$, one need only consider the subnetworks containing goal variables, and evaluate the probability of success for each of the polynomially many states of that subnetwork.

Given two Bayes nets $B_{1}$ and $B_{2}$ over such a "factored" structure $G$, and given respective policies $\pi_{1}$ and $\pi_{2}$ generated by planning algorithm $\mathcal{A}$, it is not much harder to compute

$$
\max _{s}\left|\operatorname{Succ}\left(\mathcal{A}_{1}, B_{1}, s, h\right)-\operatorname{Succ}\left(\mathcal{A}_{2}, B_{2}, s, h\right)\right|
$$

than to compute $\operatorname{Succ}\left(\mathcal{A}_{i}, B_{i}, s, h\right)$ for any particular $s$. We need only compute, for individual subnetwork $C_{i}^{j}(i \in\{1,2\})$ over structure $G^{j}, \max _{s} \mid \operatorname{Succ}\left(\mathcal{A}_{1}, C_{1}^{j}, s, h\right)-$ $\operatorname{Succ}\left(\mathcal{A}_{2}, C_{2}^{j}, s, h\right) \mid$. If any of these values is greater than a given $\theta$, then $\max _{s}\left|\operatorname{Succ}\left(\mathcal{A}_{1}, B_{1}, s, h\right)-\operatorname{Succ}\left(\mathcal{A}_{2}, B_{2}, s, h\right)\right|>\theta$, and $\left\langle\mathcal{A}, B_{1}, B_{2}, h\right\rangle \notin S B P l a n$.

\section{Related Work}

\section{Sensitivity Analysis}

The most common notion of robustness is an insensitivity to variation of one or more parameters in a network. One technique for gaining insight into this notion of robustness is sensitivity analysis. The basic idea is to systematically vary one or more of the conditional probability values of the Bayes net and to study the resultant effects on the output ${ }^{15}$. One recent development, SAMIAM ${ }^{2}$, is an implemented tool for actually computing the sensitivity of one parameter to another. Varying each probability in the network individually while studying each of the individual effects on the output is called one-way sensitivity analysis. In an $n$-way sensitivity analysis, $n$ of the probability assessments are varied simultaneously, which demonstrates each of the individual effects of varying each of the $n$ probabilities and also reveals joint or synergistic effects.

Unfortunately, the brute-force approach to one-way sensitivity analysis of a Bayes net is computationally time consuming, since for each probability value under study, a number of propagations must be investigated where each propogation requires computing the output from the network. Thus, using this approach, the computational burden for even a one-way sensitivity analysis can be prohibitive even for a small Bayes net ${ }^{3}$.

Laskey was the first to address the computational complexity of sensitivity analysis of Bayes nets. She introduced a method for using the partial derivative of probability values to yield a first-order approximation of the effect of varying a single probability parameter. While her method requires considerably less computation 
than the brute-force approach, it provides insight only in the effect of a small variation of a probability; a larger variation will rapidly break the technique down 15. This has been extended to consider "admissable deviation" of parameters by Renooij and van deer Gaag ${ }^{32}$. However, their work does not consider planning.

Undertaking a complete one-way sensitivity analysis is a computationally difficult task for non-trivial problems. Instead of it, attention has been directed to using the graphical structure of the network in order to determine independences of the output on probabilities in the network. This information is then used to eliminate the varying of probabilities that will not change the output ${ }^{3}$. Some recent progress has also been made in developing a methodology for $n$-way sensitivity analysis which requires fewer outward propagations of the network to determine upper and lower bounds on the probabilities ${ }^{14}$.

However, Henrion et al. have found that diagnostic performance with Bayesian belief networks is often surprisingly insensitive to imprecision in the numerical probabilities ${ }^{10}$. Therefore it makes sense to consider how the probability data is obtained in a given application before choosing a technique for evaluating the robustness of the output. In the case when two or more experts are consulted to determine probabilities, their estimates of each of the probabilities in the Bayes net may differ, giving two different Bayes nets on the same graph. This leads us to the problem considered in this paper: deciding whether those differences matter to the planner.

The notion of robustness in the sensitivity analysis literature that is closest to ours is that of Pradhan, et al., who consider the experimental effects of varying all of the parameters at once ${ }^{27}$. This is called uncertainty analysis by Kjælrulff and van der Gaag ${ }^{14}$ and is also considered by Henrion, et al. ${ }^{10}$. Our work differs from theirs in three key aspects: we consider planning, rather than inference, and we introduce planning into the problem explicitly; we consider the algorithmic complexity of determining robustness, and we compare two explicit sets of probabilities, rather than varying one set.

\section{Integration of Probabilistic Information}

The problem of integrating probabilistic information has been considered by researchers in a variety of different fields of computer science and statistics. As mentioned in Section 1, the largest body of work on the fusion of uncertain data comes from the area of multisensor fusion (see ${ }^{1,9}$ for early summaries). The basic problem addressed in this field is similar to ours: a number of sensors provides the user/reasoning agent with a set of data streams carrying uncertain data which need to be combined together. For example, Rehg, Murphy, and Fieguth ${ }^{28}$ study the problem of detecting whether a human, watched by four independent sensors, is speaking. Each sensor watches for particular features on the human face (face detection, skin color detection, skin texture, mouth motion) and the output of each sensor is a Bayes net. The reasoning agent then combines (fuses) the nets into one that allows it to detect whether a human is speaking. This work has been extended 
recently by Pavlovic et al. ${ }^{25}$.

Different methods of fusion of probabilistic information have been proposed. Most of them fall into one of the two categories: (i) integration techniques for specific problems and (ii) so called "toolbox" approaches. The multisensor fusion work mentioned above belongs to the first category: the solutions proposed in ${ }^{28,25}$ are specific to the particular problem being investigated and to the particular sensors being used. Other examples of this approach include the work of Druzdzel and Diez

${ }^{6}$ and the power prior approach of Thurston and Ibrahim ${ }^{30}$. Laskey and Mahoney ${ }^{16}$ propose to use on-line learning for integration of probabilistic knowledge. In ${ }^{6}$ the problem of combining the knowledge from different sources when building Bayes nets is considered. This problem is similar to our. Druzdzel and Diez gave a detailed analysis of the integration problem for a particular Bayesian model. On the other hand, our motivating question is "When can we skip the integration phase altogether?"

An example of a "toolbox" approach is the work of Dekhtyar, Ross, and Subrahmanian on combining probabilities in Temporal Probabilistic databases ${ }^{4}$. They introduce a special compaction operation whose purpose is to combine the probabilities from different probability distributions. This operation is parameterized by the combination function that dictates how the probability integration should proceed. Users are allowed to define their own combination functions to be used in the system. While more flexible, this approach factors the problem of developing the actual integration methods out of the Temporal Probabilistic database framework.

\section{Discussion}

The question of whether two sets of probability distributions for a Bayes net structure are equivalent (in any sense) is a natural one, and one that anyone building Bayes nets must address at some point. We have considered equivalence of Bayes nets with respect to planning. Although this question has been addressed in terms of sensitivity analysis, we are not familiar with any work that compares entire sets of conditional probability tables at once in the context of planning. This might be explained by our results, namely, that it is computationally infeasible in the general case to do so. However, Section 6 offers the first glimmerings of hope that this problem might in fact be tractable for some cases that occur in real life.

Because more and more heuristics for NP- and coNP-complete problems are being developed, as well as some heuristics for $\mathrm{NP}^{\mathrm{PP}}$-complete problems ${ }^{19}$, there is an additional hope that the equivalence problems described here might be approached heuristically. Future work could include an extension of the results in Section 6, heuristics for these equivalence problems, and further consideration of the notion of equivalence with respect to planning for Bayes nets. 


\section{Acknowledgements}

The work of the second author was partially supported by NSF grant CCR-0100040. The authors would like to thank the anonymous reviewers for suggesting ways to improve the paper. We are also grateful to Jesse Hoey for providing us with useful information about SPUDD.

1. M.A. Abidi and R.C. Gonzalez, editors. Data Fusion in Robotics and Machine Intelligence. Academic Press, Inc., San Diego, CA, 1992.

2. Hei Chan and Adnan Darwiche. When do numbers really matter? In Proc. Uncertainty in $A I$, pages $65-74,2001$.

3. Veerle M.H. Coupe and Linda C. van der Gaag. Practicable sensitivity analysis of bayesian belief networks. In M. Huskova, P. Lachout, J.A. Visek. Prague Stochastics '98 - Proceedings of the Joint Session of the 6th Prague Symposium of Asymptotic Statistics and the 13th Prague Conference on Information Theory, Statistical Decision Functions and Random Processes, Union of Czech Mathematicians and Physicists, pages $81-86,1998$.

4. Alex Dekhtyar, Robert Ross, and V.S. Subrahmanian. Probabilistic temporal databases, i: Algebra. ACM Transactions on Database Systems, 26(1):41-95, 2001.

5. Marek J. Druzdzel and F. Javier Diez. Criteria for combining knowledge from different sources in probabilistic models. In Working Notes, Workshop on Fusion of Domain Knowledge with Data For Decision Support, UAI '00, pages 23-29, 2000.

6. Marek J. Druzdzel and F. Javier Diez. Criteria for combining knowledge from different sources in probabilistic models. In Working Notes, Workshop on Fusion of Domain Knowledge with Data For Decision Support, UAI '00, pages 23-29, 2000.

7. Scott Ferson, Vladik Kreinovich, L. Ginzburg, K. Sentz, and D.S. Myers. Constructing probability boxes and Dempster-Shafer structures, 2002.

8. Richard Fikes and Nils J. Nilsson. STRIPS: A new approach to the application of theorem proving to problem solving. Artificial Intelligence, 2(3 and 4), 1971.

9. D. Hall. Mathematical Techniques in Multisensor Data Fusion. Artech House, Boston, MA, 1992.

10. Max Henrion, Malcolm Pradhan, Brendan Del Favero, Kurt Huang, Gregory Provan, and Paul O'Rorke. Why is diagnosis using belief networks insensitive to imprecision in probabilities? In Proc. 12th Conference on Uncertainty in AI, pages 307-314, 1996.

11. Jesse Hoey, Robert St-Aubin, Alan Hu, and Craig Boutilier. SPUDD: Stochastic planning using decision diagrams. In Proceedings of the Fifteenth Conference on Uncertainty in Artificial Intelligence, pages 279-288, Stockholm, Sweden, 1999.

12. Jesse Hoey, Robert St-Aubin, Alan Hu, and Craig Boutilier. SPUDD: Stochastic planning using decision diagrams. In Proceedings of the Fifteenth Conference on Uncertainty in Artificial Intelligence, pages 279-288, Stockholm, Sweden, 1999.

13. Jesse Hoey, Robert St-Aubin, Alan Hu, and Craig Boutilier. Optimal and approximate stochastic planning using decision diagrams. Technical Report TR-00-05, University of British Columbia, 2000.

14. Uffe Kjærulff and Linda C. van der Gaag. Making sensitivity analysis computationally efficient. In Proc. Uncertainty in AI, pages 317-325, 2000.

15. Kathryn Blackmond Laskey. Sensitivity analysis for probability assessments in bayesian networks. IEEE Transactions on Systems, Man, and Cybernetics, 25(6):136$142,1995$.

16. Kathy Laskey and Suzanne Mahoney. Knowledge and data fusion in probabilistic networks, 2002. Manuscript. 
17. Michael L. Littman. Probabilistic propositional planning: Representations and complexity. In Proceedings of 14 th National Conference on Artificial Intelligence. AAAI Press / MIT Press, 1997.

18. Michael L. Littman, Judy Goldsmith, and Martin Mundhenk. The computational complexity of probabilistic plan existence and evaluation. Journal of AI Research, 9:1-36, 1998.

19. Michael L. Littman, Stephen M. Majercik, and Toniann Pitassi. Stochastic Boolean satisfiability. Journal of Automated Reasoning, 27(3):251-296, 2000.

20. M. Majercik and Michael L. Littman. MAXPLAN: a new approach to probabilistic planning. In Artificial Intelligence and Planning Systems, pages 86-93, 1998.

21. Stephen M. Majercik and Michael L. Littman. Using caching to solve larger probabilistic planning problems. In Proceedings of Fifteenth National Conference on Artificial Intelligence, pages 954-959, 1998.

22. Pedrito Maynard-Zhang and Daniel Lehmann. Representing and aggregating conflicting beliefs. Journal of Artificial Intelligence (JAIR), 19:155-203, 2003.

23. Martin Mundhenk, Judy Goldsmith, Christopher Lusena, and Eric Allender. Complexity results for finite-horizon Markov decision process problems. Journal of the ACM, 47(4):681-720, 2000.

24. Christos H. Papadimitriou and John N. Tsitsiklis. The complexity of Markov decision processes. Mathematics of Operations Research, 12(3):441-450, 1987.

25. V. Pavlovic, A. Garg, J. Rehg, and T. Huang. Multimodal speaker detection using error feedback dynamic Bayesian networks. In Computer Vision and Pattern Recognition, volume 2, pages 34-41, 2000.

26. Judea Pearl. Probabilistic Reasoning in Intelligent Systems: Networks of Plausible Inference. Morgan Kaufmann Publishers, San Mateo, California, 1988.

27. Malcolm Pradhan, Max Henrion, Gregory Provan, Brendon del Favero, and Kurt Huang. The sensitivity of belief networks to imprecise probabilities: An experimental investigation. Artificial Intelligence, 85(1-2):363-397, 1996.

28. James M. Rehg, Kevin P. Murphy, and Paul W. Fieguth. Vision-based speaker detection using Bayesian networks. In IEEE Conference on Computer Vision and Pattern Recognition, pages 110-116, Ft. Collins, CO, 1999.

29. Pedrito Maynard Reid II and Ursula Chajewska. Aggregating learned probabilistic beliefs. In Proc. Uncertainty in Artificial Intelligence (UAI '01), number 17, pages 354-361, 2001.

30. Sally W. Thurston and Joseph G. Ibrahim. Informative prior specification for linear regression models using parameter decompositions, 2001.

31. Jacobo Torán. Complexity classes defined by counting quantifiers. Journal of the ACM, 38(3):753-774, 1991.

32. L.C. van der Gaag and S. Renooij. Analysing sensitivity data from probabilistic networks. In J. Breese and D. Koller, editors, Proc. Seventeenth Conference on Uncertainty in Artificial Intelligence (UAI '01), pages 530-537. Morgan Kaufmann Publishers, 2001. 


\section{Appendix A. Appendix: Sample SPUDD Code}

We provide here an example, in SPUDD format ${ }^{12}$ from our Horseback Riding Resort problem. The conditional probabilities are from Set 1 as described in Sectionsec:example.

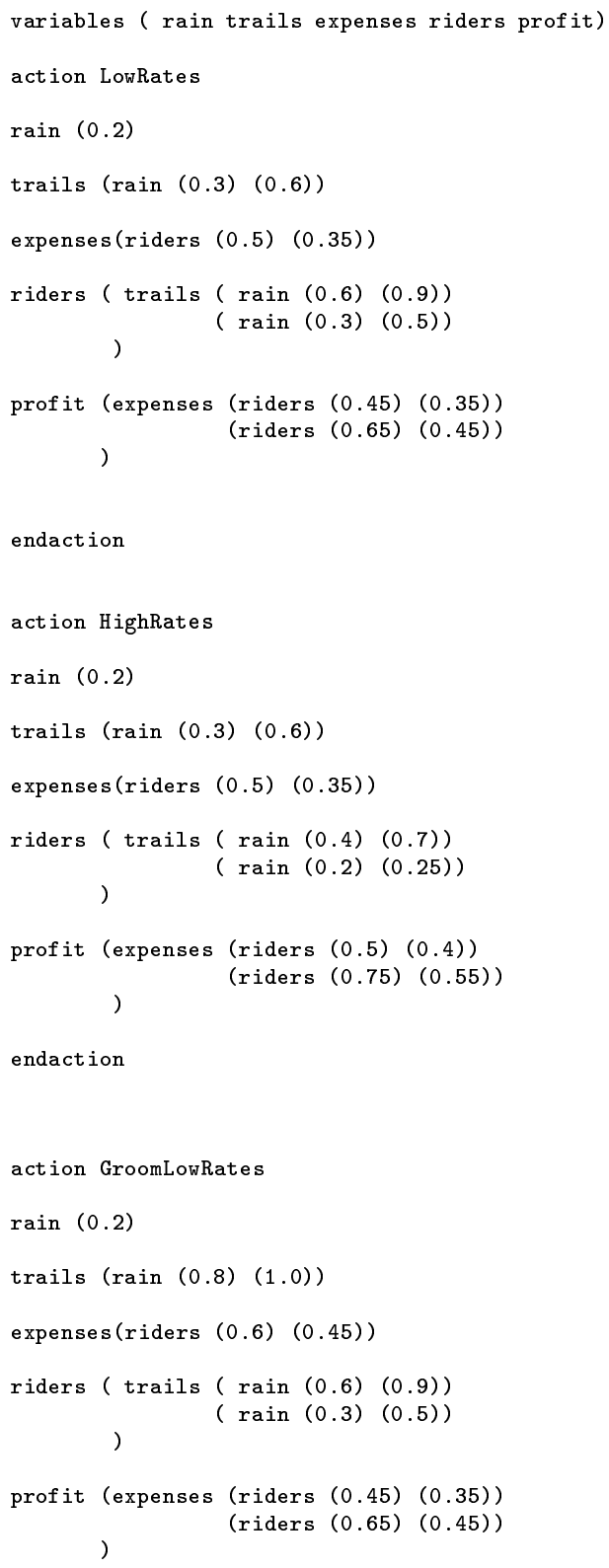




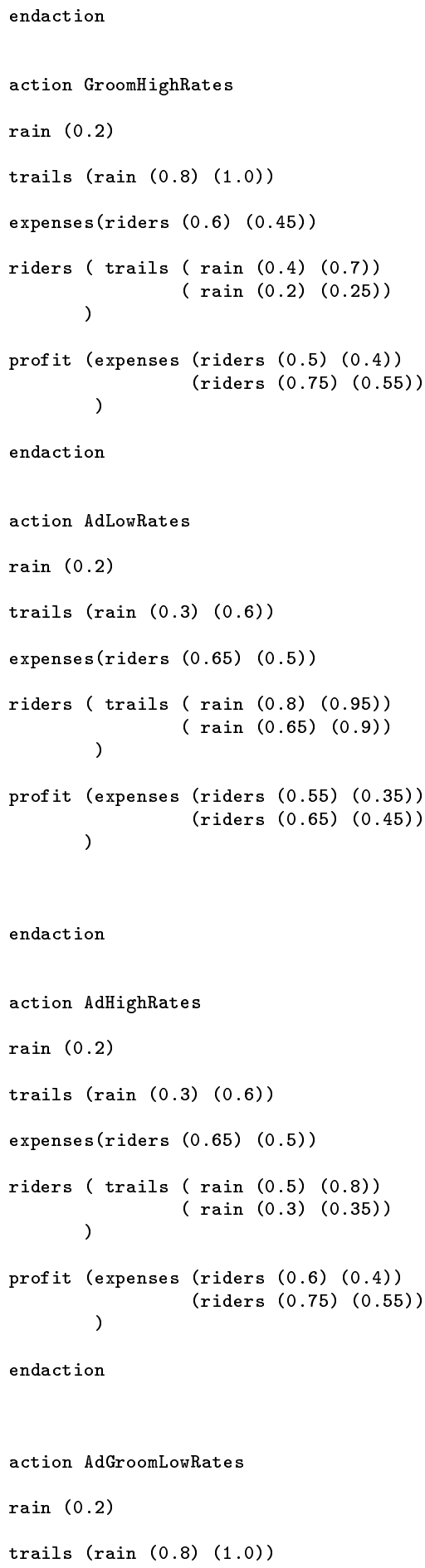




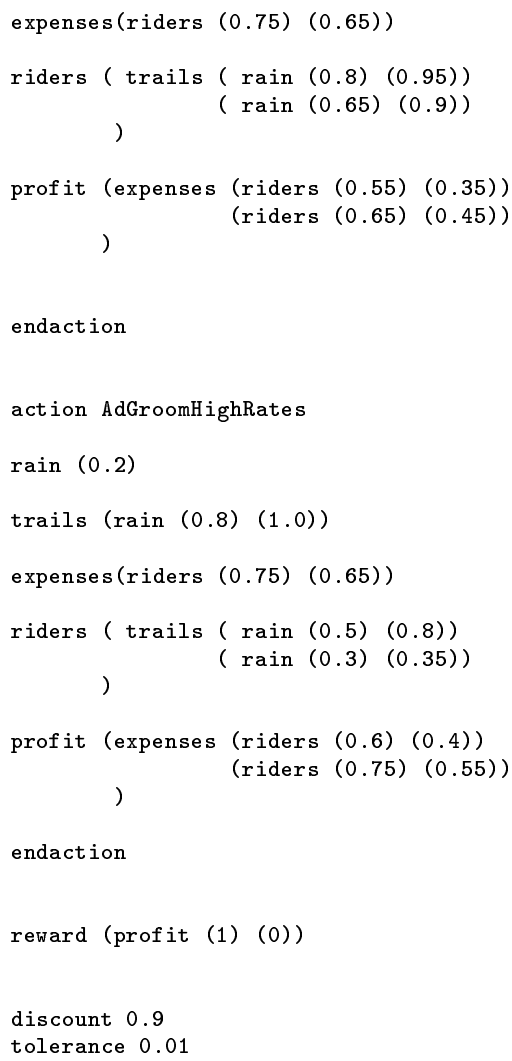

